\title{
Informationstechnik an Schulen
}

\author{
C. Schrack \\ Online publiziert am 4. September 2015 \\ (c) Springer Verlag Wien 2015
}

Die Informationstechnologie ist die zentrale Dienstleistungstechnologie in Wirtschaft und Gesellschaft. Die Nachfrage nach ITArbeitskräften ist trotz Krise ungebrochen. Rund 10.000 Personen schließen Jahr für Jahr eine Lehre, eine berufsbildende mittlere oder höhere Schule, eine Fachhochschule oder eine Universität mit diesem Schwerpunkt ab. Den größten Anteil steuern die HTLs mit einem Viertel der Absolventinnen und Absolventen bei. Nach einer Studie der IBW (Dornmayr, Helmut (2012): IT-Qualifikationen 2025) besteht gerade nach Abgängern dieser Qualifikationsstufe ("IT Experts") die größte Nachfrage in der Industrie. Diese Online-Umfrage, die unter Mitwirkung von rund 1.000 Unternehmen in Österreich durchgeführt wurde, hat auch gezeigt, dass die im Rahmen der HTLAusbildung vermittelten Inhalte gut auf die zukünftigen Arbeitsbereiche abgestimmt sind. Die Expertise an den Standorten und die enge Zusammenarbeit mit den Betrieben der Region werden zu diesem Erfolg beitragen.

Mit entsprechenden Arbeitsgemeinschaften wie der BundesARGE für Informationstechnologien (siehe Artikel Mag Schmalzl: Die Bundes-ARGE für Informationstechnologien - Think Tank und Impulsgeberin) und Tagungen wie der AINAC (siehe Artikel Dipl.Ing. Schöndorfer: Austrian International Networking Academy Conference; www.ainac.at) werden diese Aktivitäten der Schulen auch seitens des Bildungsministeriums (bmbf), Sektion Berufsbildung, gefördert.

Seit 15 Jahren gibt es Notebook-Klassen in Österreich. Informationstechnik ist somit nicht nur Bildungsinhalt, sondern kann auch als eLearning-Methode den Kompetenzerwerb in allen Bildungsbereichen und Gegenständen unterstützen.

Mit 160 Schulen nimmt rund ein Drittel der Oberstufenschulen am "eLearning Cluster" des Bildungsministeriums teil (www.elc20.com). Dazu gibt es zwei bmbf-Großveranstaltungen im Jahr, bei denen der Austausch über innovative Unterrichtsmethoden und IT-Einsatz im Unterricht stattfindet (www.edidaktik.at). Darüber hinaus veranstalten auch die Pädagogischen Hochschulen regelmäßig entsprechende eLearning-Kurse und Konferenzen, wie z. B. den eLearning-Bazar (podcampus.phwien.ac.at/ebazar/). Auch in der Mittelstufe gibt es mit eLSA ähnliche Initiativen, die aber nur ausgesuchte Schulen umfassen (elsa20.schule.at). Die nächste, große Herausforderung liegt darin, diese Innovation flächendeckend im Schulwesen zu verankern, und der Zeitpunkt scheint grundsätzlich gut gewählt.

Im Rahmenprogramm "Education \& Training 2020" startet die European Commission mit "Open up Education" ein umfassendes Vorhaben zur Verankerung von IT-Innovation im Schulwesen der Mitgliedsländer. Folgende drei Ziele sollen damit verwirklicht werden: Steigerung der Qualität des Lernens, Erhöhung der Effektivität des Unterrichtens und Sicherstellung der Chancengleichheit (www.openeducationeuropa.eu).

Um die Entwicklung an den Standorten voranzutreiben, kann es hilfreich sein, folgende vier Stoßrichtungen anzudenken:

1. Förderung des Einsatzes von "digitalen Lernbegleitern" im Unterricht
2. Entwicklung von digitalen Bildungsmedien

3. Ausbau der digitalen Infrastruktur

4. Steigerung der digitalen Kompetenz von Lernenden und Lehrenden

Einerseits werden die Schulen durch die angesprochenen eLearning-Veranstaltungen und Schulentwicklungsprogramme bei der Weiterentwicklung des Unterrichts unterstützt. Anderseits fördert die Kooperation mit der u:book-Plattform der Universität Wien den gemeinsamen Ankauf der Geräte durch die Eltern (www.ubook.at). Die HTLs waren mit "Bring your own device" (BY$\mathrm{OD})$ von Anfang an Pioniere in dieser Frage des Einsatzes digitaler Lernbegleiter.

Das Angebot an digitalen Bildungsmedien hat sich durch urheberrechtliche Bedenken bisher nur verhalten entwickelt. Auf dem Weg zu neuen Geschäftsmodellen scheint es nun seitens der Anbieter ein Umdenken zu geben. Weiters soll die Entwicklung von urheberrechtlich besser zugänglichem Material wie "Open Educational Resources" europaweit massiv gefördert werden.

Der Ausstattungsgrad an digitaler Infrastruktur fällt in den Entscheidungsbereich der Schulstandorte (Schulautonomie). Die letzte Infrastrukturerhebung bescheinigt den Oberstufenschulen eine hohe Anzahl von IT-Arbeitsplätzen im europäischen Vergleich (bmbf 2012; IT-Infrastrukturerhebung). Seitens des Bildungsministeriums, Bereich IT werden unterstützende zentrale Services und Internet Packages angeboten. Weiters werden die Schulen mit dem kommenden Schuljahr durch 120 zusätzliche Systembetreuerinnen und -betreuer unterstützt.

Die Steigerung der digitalen Kompetenz von Lernenden und Lehrenden ist gleichzeitig Voraussetzung wie Ziel der Initiative. Für den Austausch der Lehrenden werden daher die angesprochenen unterschiedlichen Formate angeboten. Im Gegensatz zur herkömmlichen Weiterbildung kann es bei Schulentwicklungsprogrammen zum wünschenswerten interdisziplinären Austausch kommen.

Nichts auf der Welt ist so mächtig, wie eine Idee deren Zeit gekommen ist. (wird Victor Hugo 1802-1885 zugeschrieben). Ist die Zeit für den umfassenden IT-Einsatz an Schulen bereits gekommen? Lassen wir uns überraschen!

Schrack, Christian, MR Ing. Mag., Bundesministerium für Bildung und Frauen, IT \& eLearning in der Berufsbildung, Minoritenplatz 5, 1014 Wien, Österreich (E-Mail: ministerium@bmbf.gv.at; christian.schrack@bmbf.gv.at) 